\title{
AN ARTIFicial ImMUne SySTEM For Human- COMPUTER INTERACTION THROUGH SPEECH
}

\author{
Antara Malakar ${ }^{1}$ and Tejbanta Singh Chingtham ${ }^{2}$ \\ ${ }^{1}$ Department of Computer Engineering, Sikkim Manipal Institute Of Technolgy, Majitar, \\ Sikkim, India \\ antaram2010@gmail.com \\ ${ }^{2}$ Department of Computer Engineering, Sikkim Manipal Institute Of Technolgy, Majitar, \\ Sikkim, India \\ chingthamegmail.com
}

\begin{abstract}
The biological immune system is a multilevel resistance system which has evolved over a millions of years. Artificial immune system (AIS) covers all the exertion to develop computationally intelligent systems encouraged by the principles and processes of the vertebrate immune system. AIS employs the biological immune system's traits of learning and memorizing to solve a problem. The common techniques of AIS which explains the function and behaviour of the vertebrate immune system are clonal selection algorithm, negative selection algorithm, positive selection, immune network algorithm and dendritic cell algorithm. Negative selection is an abstract model of biological immune system that concentrates on the generation of change detectors. This paper exploits the concept of artificial immune system to solve the problem of human computer interaction. The field of human computer interaction is relatively new and hence need more exploration. The vertebrate immune system has inspired the researchers to develop strong algorithms to solve complex problems.
\end{abstract}

\section{KEYWORDS}

Artificial Immune System, Human Computer Interaction, Negative Selection, Vertebrate immune system.

\section{INTRODUCTION}

Currently the utilization of biological systems in computer applications has been drawing much interest. In this paper, several concepts from artificial immune system have been extracted and applied to solve the problems related to human computer interaction. Artificial immune system is a computational field inspired by vertebrate immune system [1]. The immune system is capable of identifying and responding to harmful alien cells that is not recognized as self-cell. Depending on the type of pathogens, and the way it enters the body, the immune system uses different response mechanisms [2][3]. One way is to destroy the infected cells and the other is to neutralize the effect. In artificial immune system, the components are artificial cells which process tasks in order to identify and forbid attacks from intrusions. The artificial cells try to mould the behavior of the immune cells of human immune system [4]. Out of the various mechanisms in the immune system that are surveyed in the AIS, negative selection is still the most discussed model. Negative selection is used to provide tolerance for self cells [5]. It is used in detecting unknown antigens

DOI : 10.5121/ijics.2012.2303 
while not reacting to self cells. Despite the new models being proposed the field of AIS including negative selection is young and not well explored [6].

In this paper, an interaction model has been proposed wherein the computer is able to distinguish between a self and a non-self pattern. The process of negative selection is utilized to produce an immune cell, and apply itself to self-recognition system [5]. This holds the advantage of being able to identify many unknown antigens throughout the process. The interaction process is through speech.

The remainder of the paper is organized as follows. Section 2 contains problem definition and section 3 describes the benefits of using speech as the interaction modality. Section 4 consists of the architecture of the proposed model. Section 5 gives the association between the biological immune system and the proposed artificial immune system model. Section 6 and 7 consist of the implementation details and a brief description of it respectively. Section 8 and 9 contains results and conclusion respectively.

\section{Problem Definition}

The domain of human-computer interaction (HCI) has not achieved more success and is still in its infancy [7]. Computers are best utilized when it is interfaced with humans. Human computer interaction is the area which discovers about ways users can interact with the computers. The study of HCI consists of various challenges like dealing with direct uses of computer systems that interact with humans [8]. Some users can interact using traditional approach of keyboard and mouse as the main input device and monitor as the main output device. But few people for some reasons may not be able to use these devices for interaction and hence require special tools to do so [9]. Since speech is the oldest and most natural means of exchanging information between the humans, researchers have been consistently attempting to migrate this natural means into HCI. Speech recognition systems help the users who in one way or the other may not be able to adopt the traditional devices.

\section{SPEECH AS THE INTERACTION MODALITY}

An interface is the common boundary between two systems. The most vital character of speech interface with humans reigns in the nature of the speech [10]. Communication can take place only when there is transmission of information. Here, information is the set of messages which is common to both man and the computer [11]. Thus, if speech is the interface between man and the computer then both must have a common language and both are capable to generate and recognize speech.

The advantages of using speech as the interaction modality are as follows:

- Speech does not require of sight and light.

- It offers natural communication.

- It can be used for alert message.

- In busy missions when eyes and hands are preoccupied, speech can be used for communication.

- Speech is omni-directional.

- It requires very less amount of energy.

- Speech is much faster than other form of primitive controls. 


\section{ArChitecture}

The biological immune system is a complex system with the basic goal to search and kill or neutralize the pathogens entering the body [3]. Lack of immune system cells creates toxic wastes inside the system which results in killing and destroying the immune system [4]. In the proposed model to preserve the natural means, intruding system has been considered as dynamically changing sets of strings. The protected system is the knowledge repository and the intruding system is the set of foreign queries given by the user which are unknown to the system. In cells of a vertebrate, the profile of expressed protein changes over time and similarly, the proposed system is subjected to different kinds of foreign cells or queries which can be viewed as non-self cells.

The proposed man-machine immune model is shown below:

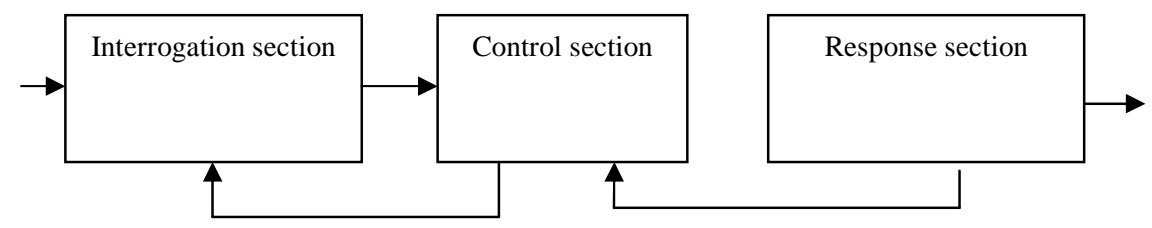

Figure1. Human computer interaction model

The recognition section is set to sense the query of the user in speech. This unit keeps on checking the dictionary for interaction failure. The main functionality of the control section is to search for the response and to update the knowledge repository. The knowledge repository which is maintained by the control section consists of universally truth queries and their actions respectively. When the control section receives the message from the recognition section, the control section analyze the information and send an action to the response section. However, in contrary if the response is not found, the response section sends a feedback to the control section. Based on the feedback, the control section updates its dictionary and uses this bit of information when that particular question is asked again.

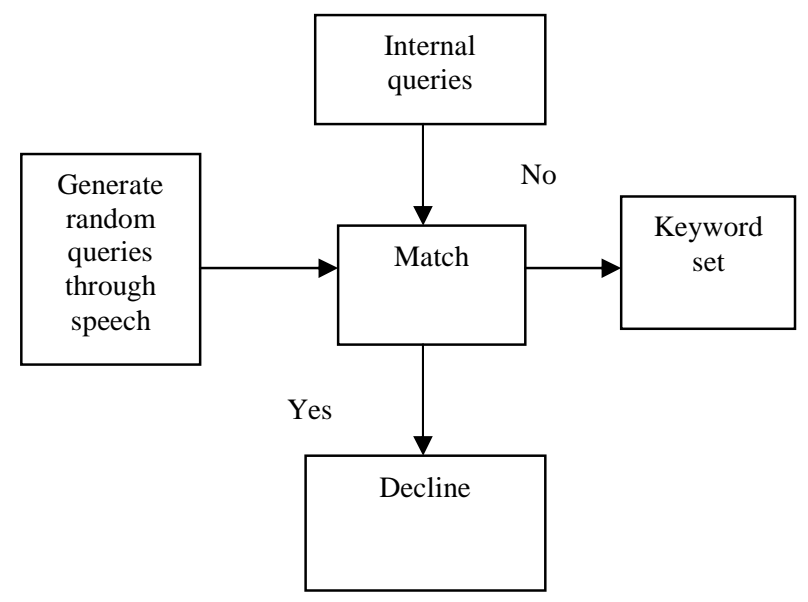

Figure2. Matching phase 
The model constitutes of the matching phase of negative selection wherein the repository consists of the queries named as internal queries. Random queries will be given in speech to the model to make out if it can be recognized or not. For this process, the random queries will be matched with the internal queries. If it is matched, that particular query will be rejected. If it is not matched, the query will be updated in the system. During the update process, the reference keyword set will be given. This keyword will be stored in the database for further detection of that query.

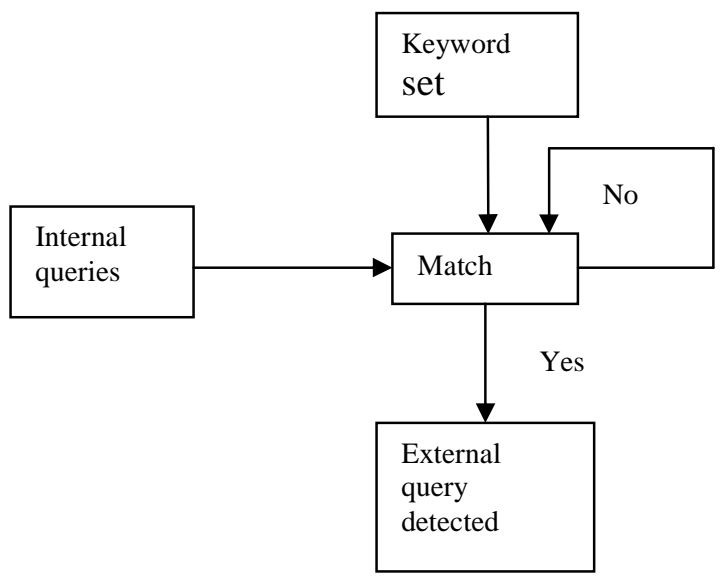

Figure 3 Detection Phase

After the matching phase, if one needs to detect the external query in negative selection, then the internal queries must be matched with the stored keywords. If it matches it is convinced that it is the external or the updated query. This detected query is the non-self cell.

\section{Association Between Human Immune Model And The Proposed SYSTEM}

\begin{tabular}{|l|l|l|}
\hline Serial Number & Human Immune System & Proposed system \\
\hline 1. & Antibody & Internal queries. \\
\hline 2. & Antigen & External queries. \\
\hline 3. & Sensitivity level & Message length \\
\hline 4. & Memory cells & Learning mechanisms \\
\hline 5. & Detector & Reference keyword. \\
\hline 6. & $\begin{array}{l}\text { Predefined amino acid } \\
\text { structure }\end{array}$ & Predefined knowledge repository \\
\hline 7. & Negative selection & Triggering \\
\hline 8. & Epitope & Condition \\
\hline
\end{tabular}


International Journal of Instrumentation and Control Systems (IJICS) Vol.2, No.3, July 2012

\begin{tabular}{|l|l|l|}
\hline 9. & Paratope & Condition \\
\hline 10. & Memory states & Size \\
\hline 11. & Traffic & Message queue \\
\hline 12. & Via keyword & Detection \\
\hline 13. & Antigen reduction & Function \\
\hline
\end{tabular}

Table 1. Matching between human immune model and the proposed model

The antibodies of human body can identify a unique part of foreign particles called antigen. In the same way the internal queries present in the knowledge repository are used to identify foreign queries.

External queries act as antigen which are the random queries given by the user. These can be detected when the internal query and the stored keyword set matches.

Sensitivity depends on the message length because as increases the sensitivity to recognize increases since error rate decreases.

After storing the queries and the keywords, if the updated query is asked in future, it will automatically give the response in speech. This learning process is optimized by successive training of the data sets.

Reference keywords are the unique identifiers to the foreign queries.

The human immune system consists of predefined amino acids. The structure of the amino acids helps to detect if it is a self or a non-self cell.

The system consists of knowledge repository which is constituted of internal queries. This is used to identify foreign queries.

If the foreign query that consists of epitopes matches with the keywords stored, then the query will be identified as unknown query and it will be included in the internal query with the unique keyword.

Paratopes can be identified when the query given matches with the internal query in which the answer can be extracted by unique keyword.

The memory states depend on the size of the knowledge repository. When the internal query and the keywords are matched, then the foreign query or the updated query is detected.

The traffic in the system depends on the size of the message queue. Keyword plays a vital role in detection of queries.

The queries stored can be monitored by a function in which existed keyword and the new keyword will be matched. If it is matched, it means that the foreign query is already present. If it does not match, then that particular query and the reference keyword is stored. In this way, the size of the query database can be reduced. 
International Journal of Instrumentation and Control Systems (IJICS) Vol.2, No.3, July 2012

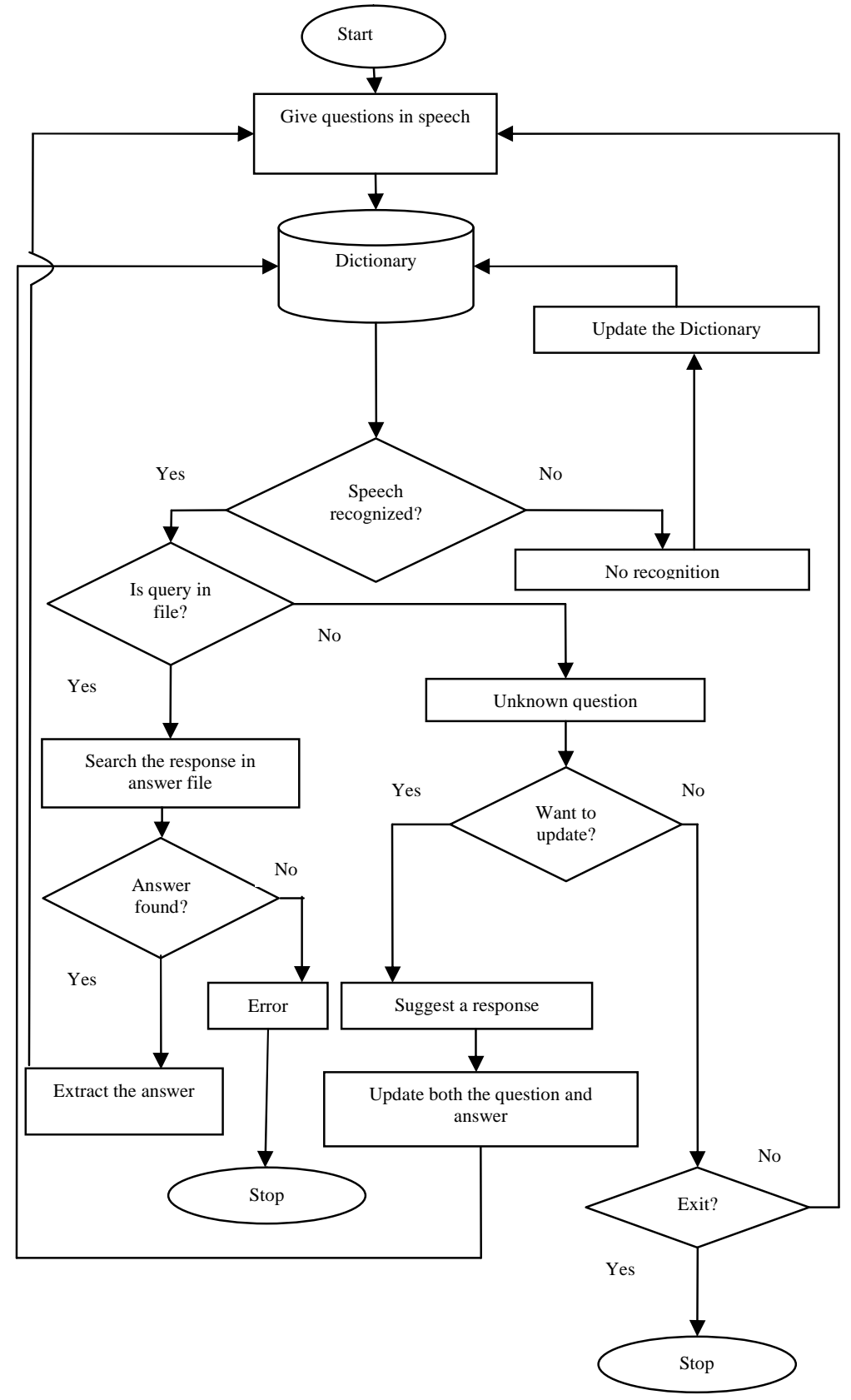

Figure4. Flowchart of the proposed model 
International Journal of Instrumentation and Control Systems (IJICS) Vol.2, No.3, July 2012

\section{IMPLEMENTATION}

The algorithm for functions to run the model is described as follows. The implementation is coded using Microsoft Visual Studio 6 [12], [13].

\section{START OF SPEECH RECOGNITION \\ Step 1: Detect query in speech \\ Step 2: Begin query check \\ - Convert speech to string \\ - Retrieve queries from query dictionary \\ - Compare string with query dictionary}

Step 3: If the string found in the query dictionary go to step 4

Step 4: Retrieve respective answer from answer dictionary

- Open answer dictionary

- Search for respective keywords

- Retrieve string followed by the keyword

Step 5: If it is not the end of speech recognition go to step 1

Step 6: If the string not found in the query dictionary go to step 7

\section{START OF DICTIONARY UPDATE}

Step 7: Update query dictionary

Step 8: Prompt for respective answer

Step 9: Update answer dictionary

- Create a unique keyword

- Update given answer in answer dictionary followed by the unique keyword

END OF DICTIONARY UPDATE

Step 10: If it is not the end of speech recognition go to step 1

END OF SPEECH RECOGNITION

\section{Description Of The Algorithm}

In the proposed model, the system will accept queries from the environment as speech through "Microsoft English Recognizer v5.1". The system will detect the query given in speech and will convert the speech into string. To check the query, whether it is known or unknown, the system will retrieve the queries, which are already stored in the dictionary of the system. The system will check if the given query is already stored in the dictionary or not. If the query is present in the query dictionary, it will detect the given query as "known query", otherwise the query will be detected as "unknown query". In the answer dictionary, every answer is stored with a unique keyword with respect to each query. For "known query", system will open the answer dictionary and will retrieve the respective answer with the unique keyword and provide the answer in the form of speech "Microsoft English Recognizer v5.1" to the environment. For "unknown query", system will update the unknown query in the query dictionary and prompt user to suggest an answer for that particular unknown query. The suggested reply will be updated in the answer dictionary with a unique keyword generated by the system. After updating both query and answer dictionary, the control s transferred to the initial stage. The process, stated above, will continue until user wants to quit from the application. 
International Journal of Instrumentation and Control Systems (IJICS) Vol.2, No.3, July 2012

\section{Result And Analysis}

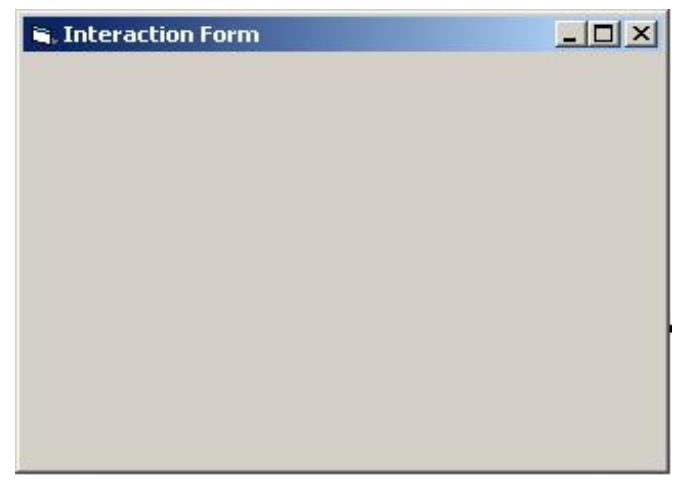

Figure5. Interaction form

At the initial phase, "Interaction form" is loaded by default. Through this form, program interacts with user through speech. In this step, any query given by user is recognised by the system.

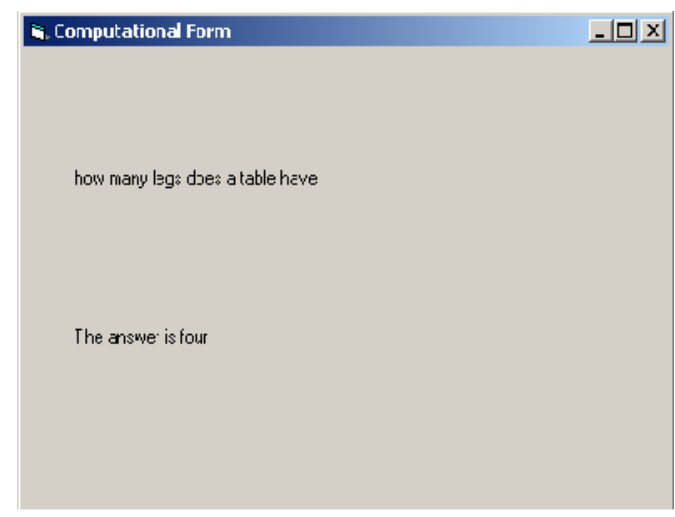

Figure6. Computational form

After recognizing the query, the system will compute if the query is a known query or not through "Computational form". Figure 6 is for a known query. The answer will be given through speech as well as text label in the form. After analysing the query the control of the system goes back to "Interaction form".



Figure7. Initial form 
International Journal of Instrumentation and Control Systems (IJICS) Vol.2, No.3, July 2012

Figure 7 is for unknown query. The query is recognized by the "Interaction" form.

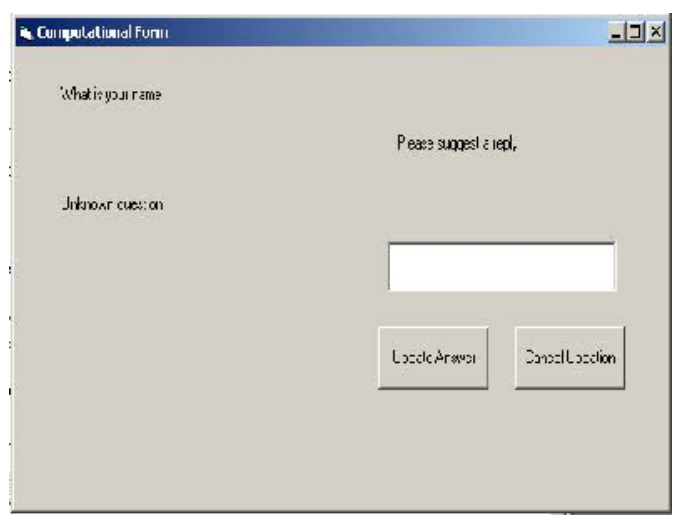

Figure8. The system acts on unknown query.

For unknown query, "Computational" form prompts for a reply from user, and a text box becomes enabled to provide the suggested reply.

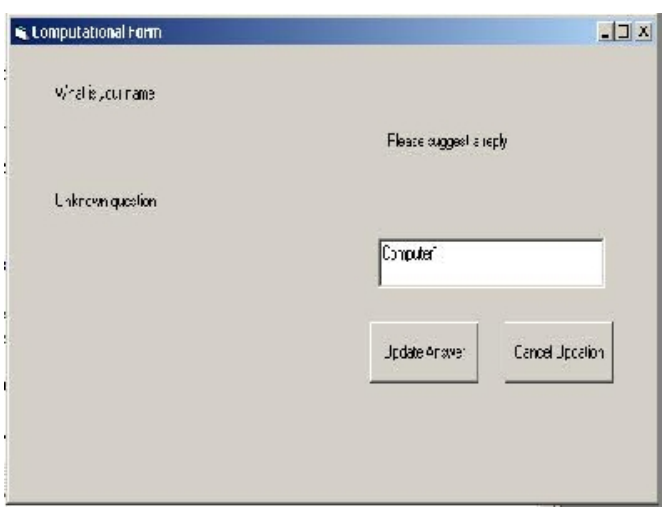

Figure9. User interacts with the system for update.

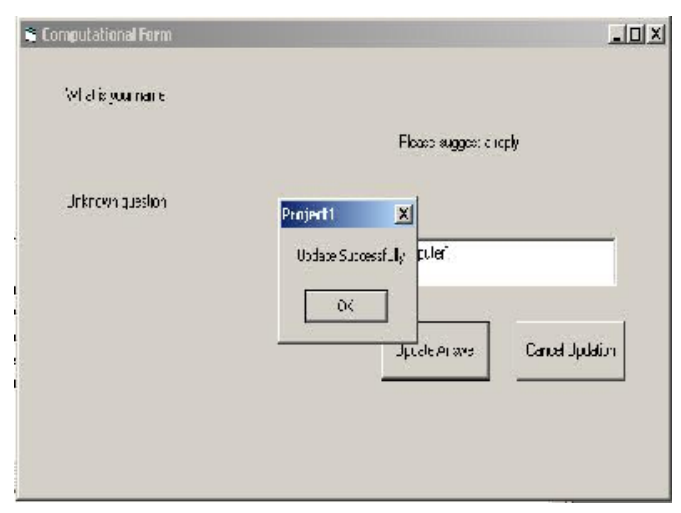

Figure10. System prompt for successful update. 
In figure 9 and 10, the answer suggested by the user in the text box, will be updated by clicking on "Update Answer" labelled button and control goes back to "Interaction" form. User may cancel updating by clicking "Cancel Updation" button.

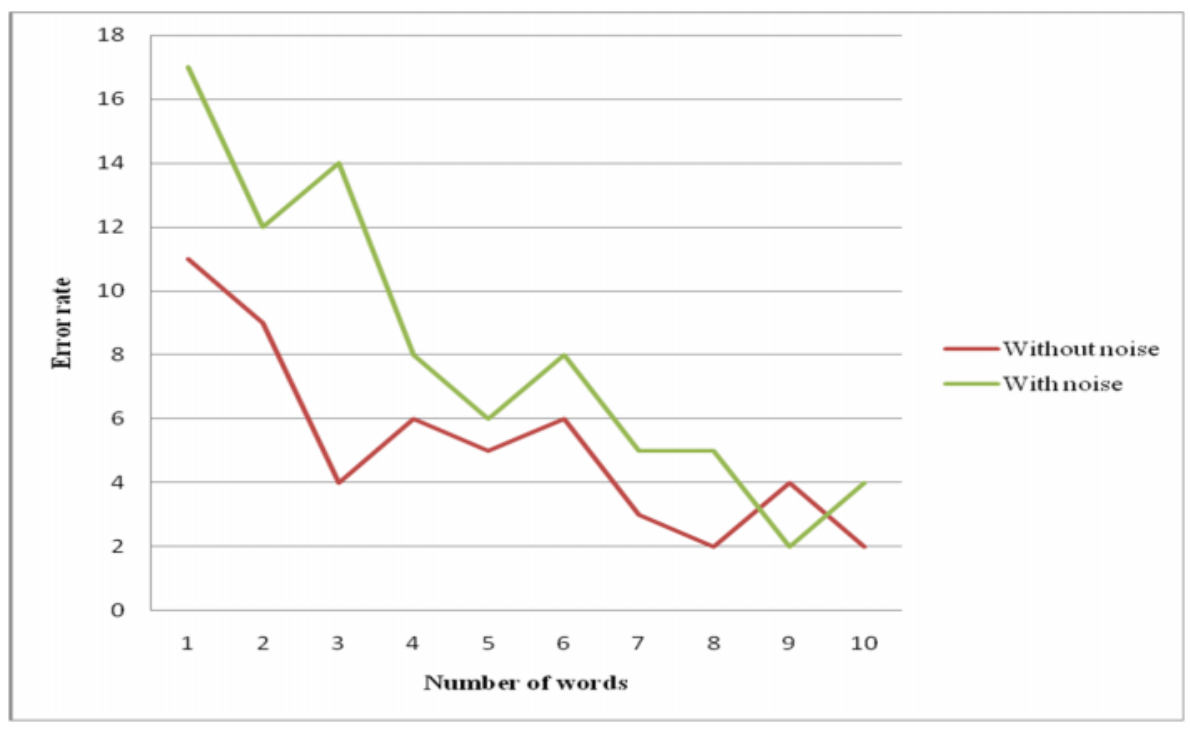

Figure11. Error rate versus number of words to find error rate with both noise and without noise.

As the length of the word increases, the error rate and the false recognition decreases. This results in quicker and better speech recognition. When speech recognition is compared between the environment with and the environment without noise, it is seen that the error rate is higher in the environment with noise as seen in figure 11 .

\section{CONCLUSION}

In this paper, human computer interaction through speech inspired by natural immune system is presented. The proposed human computer interaction model has been designed by mimicking the principles of immune system. Initially the system is trained for a particular set of queries through speech and gradually it learns the traits of foreign queries. During the speech recognition, the system was subjected to environmental noise. From the result it can be analyzed that the error rate is much lower when the system is devoid of noise.

\section{REFERENCES}

[1] Tejbanta Singh Chingtham and Shivashankar B. Nair, 'An Artificial Immune System For A Multiagent Robotics System', World Academy Of Science, Engineering and Technology, 2005.

[2] Hofmeyr S.A. and Forrest S., 'Immunity by Design: An Artificial Immune System', Proceedings of Genetic and Evolutionary Computation Conference, 1999.

[3] R.Challoo, P.Rao, S.Ozcelik, L. Challoo, S.Li, 'Navigation Control and Path Mapping of a mobile robot using artificial immune system', International Journal of Robotics and Automation, Volume (1), Issue (1).

[4] J.R.Al-Enezi, M.F. Abbod, S.Alsharhan, 'Artificial Immune Systems-Models, Algorithms And Applications', IJRRAS 3(2), May 2010.

[5] Xiaoshu Hang and Honghua Dai, 'Applying both Positive and Negative Selection to Supervised Learning for Anomaly Detection’, GECCO’05, June 25-29, Washington, D.C., USA. 
International Journal of Instrumentation and Control Systems (IJICS) Vol.2, No.3, July 2012

[6] Kwee-Bo Sim and Dong-Wook Lee 'Modelling of Positive Selection for the development of a Computer Immune System and a Self-Recognition Algorithm', International Journal of Control, Automation and Systems Vol.1, No. 4 , December 2003.

[7] Jonathan Grudin, 'Three Faces of Human-Computer Interaction', IEEE Annals of the History of Computing, 1998.

[8] F.Karrey, M. Alemzadeh, J.A.Saleh and M.N.Arab (2008), 'Human-Computer Interaction: Overview on State of the Art', International Journal on Smart Sensing and Intelligent Systems, Vol.1, March 2008.

[9] Rajeev Sharma and Thomas S. Huang, 'Toward Multimodal Human Computer Interface', Proceedings of the IEEE, Vol.86, May 1998.

[10] Roger K. Moore, 'Towards Speech-Based Human-Robot Interaction', Proc. Symposium on Language and Robotics, Aveiro, Portugal, 2007.

[11] Alejandro Jaimes and Nicu Sebe, 'Multimodal Human Computer Interaction: A Survey', IEEE International Workshop on Human Computer Interaction, China, 2005.

[12] Noel Jerke, The Complete Reference Visual Basic 6, Tata McGraw-Hill Edition 1999, 22nd reprint 2005.

[13] Tejbanta Singh Chingtham, G.Sahoo, M.K.Ghose (2010), 'An Unmanned Aerial Vehicle as HumanAssistant Robotics System', IEEE International Conference on Computational Intelligence and Computing Research 2010.

\section{Authors}

Short Biography

Antara Malakar is an M.Tech student in Sikkim Manipal Institute of Technology, Sikkim, India, in Department of Computer Science and Engineering. She received her B.E. degree from Visveswaraiah Technological University, Karnataka, India in Department of Computer Science and Technology.

Tejbanta Singh Chingtham is associated with Sikkim Manipal Institute of Technology since August, 2000 and is working as an Associate Professor in the Department of Computer Science \& Engineering. He received his B.Tech in Computer Science \& Engineering from Bharathiar University, Coimbatore, India, M.Tech from Indian Institute of Technology Guwahati, India in Computer Science \& Engineering and $\mathrm{PhD}$ from Birla Institute of Technology Mesra, Ranchi, India. He is a member of IEEE, IAENG and IACSIT. He has served as Technical Committee Members of Various

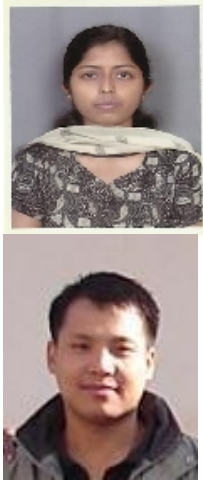
Journals and Conferences. 